\title{
Estimulação da sucção não nutritiva na "mama vazia" em bebês prematuros: relato de casos
}

\author{
Non-nutritive sucking stimulation on the "emptied breast" in \\ preterm infants: case report
}

\author{
Crisiane Venson ${ }^{1}$, Cristina Ide Fujinaga ${ }^{2}$, Gilsane Raquel Czluniak ${ }^{3}$
}

\begin{abstract}
RESUMO
Atualmente, o leite materno é o principal alimento a ser oferecido ao pré-termo segundo as recomendações de organizações nacionais e internacionais de proteção, apoio e incentivo ao aleitamento materno. O uso de mamadeiras e chupetas é desaconselhável por provocar confusão de bicos e aumentar o desmame precoce. A literatura aponta a sucção na "mama vazia" como um método alternativo de estimulação da sucção não nutritiva em prematuros. $\mathrm{O}$ objetivo deste relato de casos é descrever a suç̧ão na "mama vazia" em prematuros quanto ao tempo de utilização de sonda gástrica, tempo de internação hospitalar, ganho de peso e prevalência do aleitamento materno exclusivo na alta hospitalar. Trata-se de estudo de caso. A amostra foi composta por 19 prematuros com idade gestacional média de 32 semanas assistidos na Unidade de Cuidados Intensivos da instituição de origem, que receberam a estimulação na "mama vazia". Quando a mãe estava ausente, foi realizada a estimulação com dedo mínimo enluvado. Para análise dos dados, utilizou-se estatistica descritiva. Verificou-se que os prematuros permaneceram em média 22 dias utilizando a sonda gástrica. Com relação ao tempo de internação hospitalar, os bebês permaneceram em média 30 dias internados. O ganho de peso foi em média 23 gramas/dia. A prevalência do aleitamento materno na alta hospitalar foi de $100 \%$. Percebe-se que a sucção não nutritiva na "mama vazia" pode ser um método eficaz no aumento da prevalência do aleitamento materno na alta hospitalar e como uma técnica alternativa de estimulação da sucção não-nutritiva.
\end{abstract}

Descritores: Prematuro; Comportamento de sucção/fisiologia; Mama; Aleitamento materno; Ganho de peso; Relações mãe-filho

\section{INTRODUÇÃO}

Atualmente, a alimentação do pré-termo insere-se numa abordagem que abrange não só os aspectos nutricionais e de sobrevivência, mas também os que se referem ao seu desenvolvimento global e qualidade de vida, determinado pelo equilíbrio de suas necessidades biológicas, suporte ambiental e familiar. Nesse sentido, há uma constante busca de condições ou aspectos que visem favorecer a continuidade da vida do prematuro, além do acesso aos recursos humanos e tecnológicos, de forma humanizada ${ }^{(1,2)}$.

Desta forma, o aleitamento materno tem sido muito incen-

Trabalho realizado no Curso de Fonoaudiologia da Universidade Estadual do Centro Oeste - UNICENTRO - Irati (PR), Brasil.

(1) Fonoaudióloga da Associação de Pais e Amigos dos Excepcionais de São Joaquim - APAE - São Joaquim (SC), Brasil.

(2) Doutor, Professor Adjunto B do Departamento de Fonoaudiologia da Universidade Estadual do Centro-Oeste - UNICENTRO - Irati (PR), Brasil. (3) Mestre, Professora Assistente A do Departamento de Fonoaudiologia da Universidade Estadual do Centro-Oeste - UNICENTRO - Irati (PR), Brasil. Endereço para correspondência: Cristina Ide Fujinaga. R. Nossa Senhora de Fátima, 256/2, Centro, Irati (PR), Brasil, CEP: 84500-000. E-mail: cifujinaga@irati.unicentro.br

Recebido em: 9/9/2009; Aceito em: 22/3/2010 tivado na Unidade de Terapia Intensiva Neonatal ${ }^{(3,4)}$. Muitos serviços adotam ações que apóiam, incentivam e protegem o aleitamento. Uma das ações é evitar o oferecimento de chupetas e bicos artificiais, uma vez que a chance do desmame é maior entre os usuários destes bicos, pois diminuem a frequência da duração das mamadas e suspeita-se, ainda, que provocam confusão de bicos nos recém nascidos ${ }^{(5)}$.

A estimulação da sucção não nutritiva é uma forma de auxiliar na transição da alimentação gástrica para via oral, no entanto, historicamente, é realizada utilizando a chupeta ${ }^{(6)}$, contrariando assim as atuais recomendações de assistência à alimentação ao prematuro. A estimulação realizada no dedo enluvado pode ser uma alternativa para não provocar a confusão de bicos ${ }^{(6)}$ embora haja escassez de estudos que utilizaram essa técnica. Destaca-se a estimulação da sucção não nutritiva utilizando-se a "mama vazia", técnica na qual a mãe é orientada a esvaziar completamente a mama e oferecê-la ao bebê $\hat{e}^{(7)}$. Define-se como "mama vazia" a mama materna após a ordenha manual do leite, de ambas as mamas, até que não haja mais fluxo de ejeção com expressão manual. Utiliza-se o termo entre aspas admitindo-se que a mama materna não fica completamente vazia, levando-se em conta a presença do leite posterior, produzido por ação hormonal e estimulado 
pela demanda. Considera-se que essa técnica é um tipo de sucção não nutritiva pelo fato do prematuro não ser capaz de realizar uma sucção suficientemente eficaz para retirar o leite posterior da mama.

Assim, o presente relato de casos justifica-se pela necessidade de se buscar alternativas para possibilitar aos bebês pré-termo uma alimentação mais funcional e segura e direcionando-a ao seio materno, estando ciente de todas as dificuldades decorrentes da prematuridade. Acredita-se que a sucção na "mama vazia" pode ser considerada como uma técnica inovadora na assistência fonoaudiologica junto aos bebês prematuros. Em especial, a alimentação no seio materno produz grande impacto no desenvolvimento global destes bebês, acreditando-se ser de fundamental importância. A presença da estimulação da sucção não nutritiva na "mama vazia" junto a esta população proporcionará maiores chances de sucesso no aleitamento materno ${ }^{(7,8)}$.

O objetivo do presente estudo é descrever os efeitos da sucção não nutritiva na "mama vazia" em bebês pré-termo quanto ao tempo de utilização de sonda gástrica, tempo de internação hospitalar, ganho de peso e prevalência do aleitamento materno na alta hospitalar.

\section{APRESENTAÇÃO DOS CASOS CLÍNICOS}

O presente estudo foi realizado na Unidade de Terapia Intensiva Neonatal (UTIN) da Irmandade Hospital Santa Casa de Irati. A UTIN possui 11 leitos credenciados pelo Sistema Único de Saúde e um leito de internamento privado. Oferece atendimento multiprofissional, constando de médicos neonatologistas, equipe de enfermagem, fisioterapeuta, psicólogo, nutricionista, assistente social e fonoaudiólogo. Além disso, é priorizado o atendimento humanizado e voltado ao aleitamento materno, sendo que as mães têm direito a visitas em todos os horários de alimentação.

A população de estudo foi constituída de bebês pré-termo e que atendiam aos seguintes critérios de inclusão:

- idade gestacional corrigida igual ou superior a 30 semanas e menor ou igual a 36 semanas e seis dias, avaliado e registrado pela equipe médica;

- estabilidade clínica;

- ausência de deformidades faciais, de distúrbios respiratórios, cardiovasculares, gastrointestinais e neurológicos ou síndromes que impedissem ou dificultassem a alimentação oral.

A amostra foi estabelecida por conveniência, de forma não probabilística, de bebês assistidos no período de março a julho de 2007, e constituída por 19 prematuros, que foram estimulados com a sucção não nutritiva na "mama vazia". $\mathrm{O}$ número reduzido de sujeitos justifica a metodologia descritiva dos resultados já que esse tamanho amostral não permite análise estatística adequada.

Na UTIN da instituição de origem, os bebês são estimulados pela sucção não nutritiva, pelo menos duas vezes ao dia, pela equipe de enfermagem ou pelas estagiárias de fonoaudiologia de um curso de graduação. Os bebês são alimentados por leite industrializado, via sonda gástrica, preparado pela nutricionista do hospital, já que no mesmo não há banco de leite humano. Quando a mãe está presente, a mesma é orientada a esgotar a mama, realizando ordenha de forma manual, até que, dessa forma, haja esgotamento completo da mama e obtenção de quantidade de leite cru a ser oferecido via sonda gástrica para o bebê. Caso a mãe esteja ausente, o leite industrializado é oferecido e é realizada a estimulação da sucção não nutritiva pelo dedo enluvado durante dez minutos ou durante a passagem de um terço da quantidade da dieta prescrita via sonda gástrica.

No momento em que o bebê apresenta estabilidade clínica, logo após a ordenha, o mesmo é colocado no seio materno e recebe estimulação da sucção não nutritiva na "mama vazia". Tal procedimento é indicado pelo serviço de fonoaudiologia e pelo médico responsável e o prematuro permanece no seio materno por cerca de dez minutos. O leite materno cru é oferecido via sonda gástrica após a estimulação na "mama vazia".

O estudo foi encaminhado para apreciação do Comitê de Ética em Pesquisa da Universidade Estadual do Centro-Oeste (UNICENTRO), protocolado pelo número 159/06, considerado aprovado. Respeitando os aspectos éticos de pesquisas com seres humanos, conforme Resolução 196/96, foi solicitada a assinatura pela mãe do bebê do Termo de Consentimento Livre e Esclarecido. Por uma questão ética, o presente estudo de casos não contou com um grupo controle e, por isso, os resultados serão apresentados de forma descritiva, sem a aplicação de testes estatísticos para comparação dos achados.

De acordo com os dados da Tabela 1, 12 dos recém-nascidos eram do gênero feminino e sete do gênero masculino. A idade gestacional ao nascimento variou de 28 a 35 semanas, com média de 32 semanas. Cabe-se ressaltar que a idade gestacional considerada no estudo foi obtida no livro de registro de nascimento da UTIN, uma vez que havia diferentes registros em prontuário. Além disso, a idade foi calculada pela equipe médica sem um procedimento padronizado. Dessa forma, alguns bebês foram avaliados pelo método Capurro Somático, enquanto outros, a idade foi calculada pela ultrassonografia de crânio ${ }^{(9)}$.

Quanto ao peso de nascimento, a média foi de aproximadamente, 1629 gramas variando de 770 a 2335 gramas. Dessa forma, com relação à classificação quanto ao peso ${ }^{(10)}$ constatouse que 11 bebês nasceram de baixo peso, sete nasceram de muito baixo peso, enquanto apenas um nasceu de extremo baixo peso. Os dados referentes ao peso foram anotados do prontuário e aferidos pela equipe de enfermagem em balança digital comum.

O peso no início da estimulação variou de 1135 a 2110 gramas, atingindo uma média de 1582 gramas. Verificou-se também que quatro dos recém-nascidos apresentaram peso inicial no momento da estimulação inferior ao peso de nascimento, alcançando uma média de 123 gramas de diferença entre o peso de nascimento e o peso inicial, sendo que tais bebês estavam no período de perda fisiológica de peso após o nascimento. Quatro bebês tiveram ganho de peso entre o período de nascimento até o início da estimulação da sucção não-nutritiva, apresentando uma média de 209 gramas de diferença entre o peso de nascimento e o peso inicial, e apenas um bebê manteve o peso de nascimento até o período de início da intervenção fonoaudiológica.

A idade pós-natal variou de três a 44 dias, atingindo uma 
Tabela 1. Caracterização dos 19 recém-nascidos pré-termo estimulados na "mama vazia"

\begin{tabular}{|c|c|c|c|c|c|c|}
\hline Recém- nascido & Gênero & $\begin{array}{l}\text { Idade gestacional } \\
\text { ao nascimento } \\
\text { (semanas) }\end{array}$ & $\begin{array}{c}\text { Peso ao nascimento } \\
\text { (gramas) }\end{array}$ & $\begin{array}{l}\text { Peso no inicio } \\
\text { da estimulação } \\
\text { (gramas) }\end{array}$ & $\begin{array}{c}\text { Idade pós-natal } \\
\text { no início da } \\
\text { estimulação (dias) }\end{array}$ & $\begin{array}{c}\text { Idade gestacional } \\
\text { corrigida }\end{array}$ \\
\hline RN 1 & Feminino & 28 & 1250 & 1300 & 13 & $29 s$ 6d \\
\hline RN 2 & Feminino & 30 & 1695 & 1650 & 12 & $32 s 5 d$ \\
\hline RN 3 & Feminino & 33 & 2335 & 2110 & 8 & $34 \mathrm{~s} 1 \mathrm{~d}$ \\
\hline $\mathrm{RN} 4$ & Feminino & 33 & 1880 & 1745 & 8 & $34 \mathrm{~s} 1 \mathrm{~d}$ \\
\hline RN 5 & Masculino & 30 & 1375 & 1250 & 5 & $30 s 5 d$ \\
\hline RN 6 & Masculino & 32 & 1200 & 1230 & 7 & $33 s$ \\
\hline RN 7 & Masculino & 32 & 1740 & 1965 & 5 & $32 s 5 d$ \\
\hline RN 8 & Masculino & 34 & 2160 & 1985 & 10 & $35 s$ 3d \\
\hline $\mathrm{RN} 9$ & Feminino & 30 & 1290 & 1290 & 5 & $30 s 5 d$ \\
\hline RN 10 & Feminino & 33 & 1650 & 1565 & 8 & $34 \mathrm{~s} 1 \mathrm{~d}$ \\
\hline RN 11 & Feminino & 30 & 1855 & 1700 & 3 & $30 s 3 d$ \\
\hline RN 12 & Feminino & 32 & 2035 & 1860 & 3 & $32 s 3 d$ \\
\hline RN 13 & Feminino & 32 & 2015 & 1900 & 3 & $32 s 3 d$ \\
\hline RN 14 & Feminino & 33 & 1400 & 1395 & 14 & $35 s$ \\
\hline RN 15 & Feminino & 34 & 2050 & 1760 & 7 & $35 s$ \\
\hline RN 16 & Feminino & 35 & 1820 & 1720 & 3 & $35 s 3 d$ \\
\hline RN 17 & Masculino & 29 & 770 & 1300 & 44 & $35 s 2 d$ \\
\hline RN 18 & Masculino & 31 & 1245 & 1205 & 12 & $32 s 5 d$ \\
\hline RN 19 & Masculino & 31 & 1180 & 1135 & 12 & $32 s 5 d$ \\
\hline Média & & 32 & 1628,6 & 1582 & 9,5 & $33 s$ 1d \\
\hline
\end{tabular}

Legenda: $\mathrm{RN}$ = recém-nascido; $\mathrm{s}$ = semanas; $\mathrm{d}$ = dias

média de dez dias. Com relação à idade gestacional corrigida, esta obteve uma média de 33 semanas e um dia, variando de 29 semanas e seis dias a 35 semanas e três dias.

Os bebês permaneceram em média 21 dias recebendo a estimulação da sucção não nutritiva, variando de sete a 37 dias. Com relação à estimulação da sucção não nutritiva, obteve-se como média diária cinco vezes ao dia, variando de três a seis vezes ao dia. A estimulação com o dedo enluvado ocorreu na maioria dos bebês, com exceção dos recém nascidos 3 , 4 e 8 . Logo, os que receberam tal estimulação ficaram em média cinco dias sendo estimulados apenas com o dedo, obtendo uma variação de dois a 13 dias. Vale ressaltar que, os bebês eram submetidos à estimulação com o dedo enluvado quando estes já estavam estáveis clinicamente, porém a mãe não se encontrava presente (Tabela 2).

Verifica-se que todos os bebês receberam estimulação da sucção não nutritiva com a "mama vazia", obtendo uma média de 12 dias de estimulação com este método, variando de cinco a 20 dias. Sempre foi dada a preferência em se colocar assim que possível o bebê no seio materno, levando-se em conta, a todo o momento, seu estado clínico.

Seis recém nascidos, sendo eles o 1, 3, 6, 15, 16 17, 18 e 19 , receberam os dois meios de estimulação no mesmo dia, ou seja, em alguns horários tiveram estimulação com o dedo enluvado e em outras com a "mama vazia".

O tempo médio de uso de sonda gástrica do grupo estimulado foi de aproximadamente 22 dias, variando de oito a 69 dias. Verificou-se que o tempo médio de internação dos bebês foi de 30 dias, variando de 15 a 72 dias.
Para se calcular o ganho de peso, foi considerado no estudo o ganho de peso no período em que ocorreu a estimulação da sucção não nutritiva. Tal dado foi calculado a partir da diferença do peso que o pré-termo apresentava no início da intervenção fonoaudiológica com o peso que o mesmo apresentava no momento da alta. Obteve-se dessa forma, conforme descrito na Tabela 3, uma média de 527 gramas, variando de -35 a 1025 gramas. Já com relação ao ganho de peso diário, obteve-se uma variação de -5 a 37 gramas, atingindo uma média de 23 gramas diariamente, durante o período de estimulação. Tais valores foram calculados a partir do ganho de peso total obtido no período da intervenção fonoaudiológica, dividido com o tempo de permanência que estes bebês receberam a estimulação da sucção não nutritiva.

Todos os prematuros receberam alta hospitalar em aleitamento materno exclusivo.

\section{DISCUSSÃO}

Com o uso prolongado de sonda gástrica para alimentação, o bebê é privado de uma série de estímulos sensoriais (gustativos, textura, temperatura), além de ocorrer atraso no desenvolvimento sensório-motor oral, pois o recém nascido não realiza a função de sucção, o que pode comprometer a sua aceitação e adaptação à alimentação oral no seio materno posteriormente $^{(8)}$.

A partir daí, vê-se a importância de estimular a sucção não nutritiva nos recém nascidos pré-termo assim que possível, pois como não possuem uma sucção eficiente abrupta, por questões 
Tabela 2. Distribuição dos bebês pré-termo segundo tipo de estimulação da sucção não nutritiva (ESNN)

\begin{tabular}{|c|c|c|c|c|c|c|}
\hline Recém- nascido & $\begin{array}{c}\text { Período em } \\
\text { que receberam } \\
\text { estimulação (dias) }\end{array}$ & $\begin{array}{l}\text { Quantidade de } \\
\text { dias em que } \\
\text { receberam a } \\
\text { estimulação (dias) }\end{array}$ & $\begin{array}{c}\text { Média de } \\
\text { estimulação } \\
\text { diária ( } n^{\circ} \text { de vezes } \\
\text { por dia) }\end{array}$ & $\begin{array}{l}\text { ESNN com dedo } \\
\text { enluvado (dias) }\end{array}$ & $\begin{array}{l}\text { ESNN com "mama } \\
\text { vazia" (dias) }\end{array}$ & $\begin{array}{c}\text { ESNN dedo } \\
\text { enluvado + "mama } \\
\text { vazia" (dias) }\end{array}$ \\
\hline $\mathrm{RN} 1$ & 19 & 13 & 3 & 5 & 5 & 3 \\
\hline $\mathrm{RN} 2$ & 22 & 11 & 5 & 2 & 9 & 0 \\
\hline RN 3 & 7 & 8 & 6,5 & 0 & 7 & 1 \\
\hline $\mathrm{RN} 4$ & 7 & 7 & 6,5 & 0 & 7 & 0 \\
\hline RN 5 & 27 & 27 & 5 & 13 & 14 & 0 \\
\hline RN 6 & 28 & 28 & 4,3 & 7 & 17 & 4 \\
\hline RN 7 & 15 & 14 & 3,6 & 2 & 12 & 0 \\
\hline RN 8 & 16 & 15 & 5,4 & 0 & 15 & 0 \\
\hline RN 9 & 31 & 24 & 3,6 & 11 & 13 & 0 \\
\hline RN 10 & 17 & 15 & 4 & 7 & 8 & 0 \\
\hline RN 11 & 16 & 17 & 4,7 & 5 & 12 & 0 \\
\hline RN 12 & 23 & 23 & 5,3 & 3 & 20 & 0 \\
\hline RN 13 & 23 & 24 & 5,4 & 4 & 20 & 0 \\
\hline RN 14 & 23 & 22 & 4,9 & 5 & 17 & 0 \\
\hline RN 15 & 14 & 15 & 5 & 6 & 6 & 3 \\
\hline RN 16 & 18 & 17 & 4,7 & 8 & 6 & 3 \\
\hline RN 17 & 28 & 18 & 4,4 & 6 & 10 & 2 \\
\hline RN 18 & 28 & 30 & 4,5 & 6 & 16 & 8 \\
\hline RN 19 & 37 & 34 & 4 & 13 & 19 & 2 \\
\hline Média & 21 & 19 & 4,7 & 5,4 & 12,2 & 1,3 \\
\hline
\end{tabular}

Legenda: $\mathrm{RN}$ = recém-nascido; $d$ = dias; ESNN = estimulação da sucção não-nutritiva

Tabela 3. Distribuição dos bebês pré-termo do grupo estimulado quanto ao ganho de peso com relação ao peso inicial à estimulação

\begin{tabular}{|c|c|c|c|c|}
\hline Recém-nascido & $\begin{array}{c}\text { Peso inicial } \\
\text { (gramas) }\end{array}$ & $\begin{array}{l}\text { Peso final } \\
\text { (gramas) }\end{array}$ & $\begin{array}{c}\text { Ganho de peso total } \\
\text { (gramas) }\end{array}$ & $\begin{array}{c}\text { Ganho de peso diário } \\
\text { (gramas) }\end{array}$ \\
\hline $\mathrm{RN} 1$ & 1300 & 2000 & 700 & 36,8 \\
\hline $\mathrm{RN} 2$ & 1650 & 2040 & 390 & 17,7 \\
\hline RN 3 & 2110 & 2075 & -35 & -5 \\
\hline $\mathrm{RN} 4$ & 1745 & 1810 & 65 & 9,2 \\
\hline RN 5 & 1250 & 2100 & 850 & 31,4 \\
\hline RN 6 & 1230 & 2100 & 870 & 31 \\
\hline RN 7 & 1965 & 2030 & 65 & 4,3 \\
\hline RN 8 & 1985 & 2520 & 535 & 33,4 \\
\hline RN 9 & 1290 & 2000 & 710 & 22,9 \\
\hline RN 10 & 1565 & 2100 & 535 & 31,4 \\
\hline RN 11 & 1700 & 1980 & 280 & 17,5 \\
\hline RN 12 & 1860 & 2100 & 240 & 10,4 \\
\hline RN 13 & 1900 & 2200 & 300 & 13 \\
\hline RN 14 & 1395 & 2080 & 685 & 29,7 \\
\hline RN 15 & 1760 & 2290 & 530 & 37,8 \\
\hline RN 16 & 1720 & 2180 & 460 & 25,5 \\
\hline RN 17 & 1300 & 2100 & 800 & 28,5 \\
\hline RN 18 & 1205 & 2230 & 1025 & 36,6 \\
\hline RN 19 & 1135 & 2140 & 1005 & 27,1 \\
\hline Média & 1582 & 2109,2 & 526,8 & 23,1 \\
\hline
\end{tabular}

Legenda: $\mathrm{RN}=$ recém nascido 
de maturação, necessitam de um período de treinamento dos movimentos de sucção e da coordenação desta função com a respiração e deglutição. Logo, a estimulação da sucção não nutritiva visa preparar o bebê para uma sucção eficiente ${ }^{(11)}$.

A revisão da literatura aponta uma lacuna sobre a sucção não nutritiva, pois os estudos não deixam claras, no método, informações importantes de como ocorre a transição da alimentação gástrica para via oral. Historicamente, a estimulação da sucção não nutritiva ocorre com uso de chupeta e não prioriza o aleitamento materno ${ }^{(6)}$. Entretanto, a exposição de bebês amamentados a chupetas e bicos artificiais no período neonatal não tem sido recomendada, pelo risco de prejuízos ao aleitamento materno ${ }^{(12)}$, apesar de que a utilização de chupetas em prematuros menores de 34 semanas parece não ter afetado a amamentação ${ }^{(13)}$. Novos estudos devem ser realizados para completo esclarecimento da repercussão do uso da chupeta em prematuros e suas implicações na prevalência do aleitamento materno.

$\mathrm{Na}$ literatura não se encontram pesquisas nacionais para comparação dos resultados do presente estudo. Com relação ao ganho de peso, não há registros de outros estudos que tenham utilizado as mesmas técnicas de sucção não nutritiva (dedo enluvado e "mama vazia"). Em estudo internacional ${ }^{(7)}$ que utilizou a "mama vazia", não fica claro o tempo de utilização de sonda gástrica, o ganho de peso diário e a prevalência do aleitamento materno exclusivo na alta hospitalar. O referido estudo demonstrou que o tempo de internação hospitalar foi em média de 24 dias e a estimulação na "mama vazia" influenciou aumento da duração da lactação em comparação aos prematuros que não receberam esse tipo de estimulo. A diminuição do tempo de internação é de grande importância à medida que são reduzidos os gastos hospitalares e promovido o vínculo mãe-bebê, situação esta extremamente importante para a manutenção do aleitamento materno.

Outro apontamento importante refere-se ao fato do prematuro estimulado na "mama vazia" estar exposto ao leite posterior da mama. A ingestão desse leite, ainda que em pequena quantidade, pode ter facilitado o ganho de peso já a utilização de leite posterior da própria mãe, ordenhado mecanicamente, promove um aumento médio de peso da ordem de 19 gramas por dia. Tal ganho de peso pode ser justificado pelo fato do leite posterior possuir maior teor de gordura ${ }^{(14)}$.

Acredita-se que o bebê recebendo a estimulação na "mama vazia" tenha seu desenvolvimento do sistema sensório motor oral potencializado, já que este procedimento estimula a sucção não nutritiva da forma mais fisiológica possível, além de proporcionar ao prematuro os estímulos gustativos de textura, temperatura e de afetividade (contato com a mãe).

A estimulação na "mama vazia" promove o envolvimento da mãe no cuidado ao prematuro e a ordenha manual. Essas favorecem, além do vínculo mãe-filho, a manutenção da produção do leite materno e a confiança da mãe enquanto cuidadora $^{(15)}$.

A "mama vazia" é um método fisiológico de estimulação da sucção não nutritiva de fácil aplicação e que envolve a mãe no cuidado ao bebê. Na prática clínica, como no caso descrito, em serviços que não possuem banco de leite humano, a técnica da "mama vazia" e a ordenha manual da mama para obtenção do leite cru a ser oferecido por sonda gástrica pode ser realiza com orientação e apoio do fonoaudiólogo. Tal prática trás benefícios como a manutenção da lactação materna e o maior ganho de peso do prematuro. Destaca-se que a atuação do fonoaudiólogo deve voltar-se para uma assistência humanizada e preocupada com as necessidades maternas e do serviço.

Como limitações do estudo, apontamos o número reduzido de participantes e a necessidade de novos estudos para cálculo do ganho de peso promovido pela estimulação da sucção não nutritiva em "mama vazia".

\section{COMENTÁRIOS FINAIS}

Finalmente, considera-se a estimulação da "mama vazia" uma técnica importante na assistência humanizada ao prematuro e a sua família, estendendo o seu impacto na prevalência do aleitamento materno e, consequentemente, no desenvolvimento global do prematuro. $\mathrm{O}$ fonoaudiólogo pode utilizar essa técnica como uma possibilidade fisiológica de sucção não nutritiva do prematuro, visando o desenvolvimento motor oral com a inclusão da mãe no cuidado ao seu filho, promovendo o aleitamento materno nessa clientela.

\begin{abstract}
Currently, breast milk is the main nourishment to be offered to preterm infants, according to the recommendation of national and international organizations for breastfeeding protection, support and incentive. The use of bottles and pacifiers is inadvisable for causing nipple confusion and increasing early weaning. Literature indicates "emptied breast" sucking as an alternative method of non-nutritive sucking stimulation in preterm infants. The aim of this case report was to describe "emptied breast" sucking in premature infants according to duration of gastric tube feeding, length of hospital stay, weight gain and prevalence of exclusive breastfeeding at discharge. The sample consisted of 19 infants with mean gestational age of 32 weeks, assisted in the Neonatal Intensive Care Unit of the hospital where the study took place. The infants received "emptied breast" sucking stimulation. When the mother was absent, stimulation was performed with a gloved finger. Data analysis used descriptive statistics. The premature infants remained, in average, for 22 days with gastric tube feeding. The preterm infants were, in average, for 30 days in the hospital. Mean weight gain was 23 grams/day. Prevalence of breastfeeding at hospital discharge was 100\%. It was observed that non-nutritive sucking on the "emptied breast" can be an effective method to increase the prevalence of breastfeeding at hospital discharge, and as an alternative technique for stimulation of non-nutritive sucking.
\end{abstract}

Keywords: Infant, premature; Sucking behavior/physiology; Breast; Breast feeding; Weight gain; Mother-child relations 


\section{REFERÊNCIAS}

1. Aguayo J. Maternal lactation for preterm newborn infants. Early Hum Dev. 2001;65 Suppl:S19-29. Review.

2. Gaíva MAM, Scochi CGS. A participação da família no cuidado ao prematuro em UTI Neonatal. Rev Bras Enferm. 2005;58(4):444-8.

3. Nascimento MBR, Issler H. Aleitamento materno em prematuros: manejo clínico hospitalar. J Pediatr (Rio J). 2004;80(5 Supl):S163-72.

4. Braga DF, Machado MMT, Bosi MLM. Amamentação exclusiva de recém-nascidos prematuros: percepções e experiências de lactantes usuárias de um serviço público especializado. Rev Nutr. 2008;21(3):293302.

5. Victora CG, Behague DP, Barros FC, Olinto MT, Weiderpass E. Pacifer use and short breastfeeding duration: cause, consequence or coincidence? Pediatrics. 1997;99(3):445-53.

6. Caetano LC, Fujinaga CI, Scochi CGS. Sucção não-nutritiva em bebês prematuros: estudo bibliográfico. Rev Latinoam Enferm. 2003;11(2):232-6.

7. Narayanan I, Mehta R, Choudhury DK, Jain BK. Sucking on the 'emptied' breast: non-nutritive sucking with a difference. Arch Dis Child. 1991;66(2):241-4.

8. Sanches MTC. Manejo clínico das disfunções orais na amamentação. J Pediatr (Rio J). 2004;80(5 Supl)):S155-62.
9. Moraes CL, Reichenheim ME. Validade do exame clínico do recém nascido para a estimação da idade gestacional: uma comparação do escore New Ballard com a data da última menstruação e ultrasonografia. Cad Saúde Pública = Rep Public Health. 2000;16(1):83-94.

10. UNICEF. Indicadores básicos. 2000. [Internet]. [citado 2003 set 11]. Disponível em: <http://unicef.org.br>.

11. Hafström M, Kjellmer I. Non-nutritive sucking in the healthy pre-term infant. Early Hum Dev. 2000;60(1):13-24.

12. Howard CR, Howard FM, Lanphear B, Eberly S, deBlieck EA, Oakes D, Lawrence RA. Randomized clinical trial of pacifier use and bottlefeeding or cupfeeding and their effect on breastfeeding. Pediatrics. 2003;111(3):511-8.

13. Collins CT, Ryan P, Crowther CA, McPhee AJ, Paterson S, Hiller JE. Effect of bottles, cups, and dummies on breast feeding in preterm infants: a randomised controlled trial. BMJ. 2004;329(7459):193-8.

14. Slusher T, Hampton R, Bode-Thomas F, Pam S, Akor F, Meier P. Promoting the exclusive feeding of own mother's milk through the use of hindmilk and increased maternal milk volume for hospitalized, low birth weight infants (<1800 grams) in Nigeria: a feasibility study. J Hum Lact. 2003;19(2):191-8.

15. Serra SOA, Scochi CGS. Dificuldades maternas no processo de aleitamento materno de prematuros em uma UTI neonatal. Rev Latinoam Enferm. 2004;12(4):597-605. 